\title{
IgG4-related sclerosing cholangitis: not always an obvious entity
}

\author{
Magdalini Velegraki ${ }^{a}$, Emmanouil Vardas ${ }^{a}$, Christos Dervenis ${ }^{b}$, Maria Fragakia ${ }^{\text {, Pinelopi Nikolaou }}{ }^{a}$, \\ Afroditi Mpitoulia , George Kazamiasc, Athanasia Sepsad, Elpida Giannikakic, Gregorios A. Paspatis ${ }^{a}$ \\ Venizeleion General Hospital, Heraklion, Crete; Metropolitan Hospital, Athens, Greece
}

\begin{abstract}
Immunoglobulin G4-related sclerosing cholangitis (IgG4-SC) is a distinct type of cholangitis, currently recognized as a biliary manifestation of IgG4-related disease. We present a case of type 3 IgG4-SC in a patient with normal IgG4 serum levels, surgically treated for suspicion of cholangiocarcinoma. This case highlights that differentiating between isolated IgG4-SC and cholangiocarcinoma can present a challenging diagnostic dilemma.
\end{abstract}

Keywords IgG4-related sclerosing cholangitis, cholangiocarcinoma

Ann Gastroenterol 2021; 34 (2): 1-3

\section{Introduction}

Immunoglobulin G4-related sclerosing cholangitis (IgG4-SC) is a distinct type of cholangitis frequently associated with autoimmune pancreatitis (AIP) and currently recognized as a biliary manifestation of a systemic disorder termed IgG4-related disease (IgG4-RD) [1]. The characteristic features of IgG4-RD are enlargement of the affected organ(s) with lymphoplasmacytic infiltrations composed of IgG4+ plasma cells, storiform fibrosis, obliterative phlebitis and high serum IgG and IgG4 levels [2]. Here, we report a patient who presented with weight loss and elevated cholestatic enzymes. Two segmental stenoses in both the hilar hepatic duct and the common bile duct (CBD) were demonstrated, and were finally attributed to type 3 IgG4-SC, after being surgically resected on suspicion of cholangiocarcinoma.

\section{Case report}

A 70-year-old male with no significant past medical history presented in March 2019 with fatigue and an 8-kg weight loss

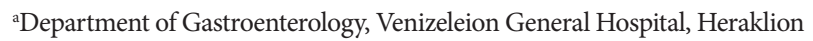
(Magdalini Velegraki, Emmanouil Vardas, Maria Fragaki, Pinelopi Nikolaou, Afroditi Mpitouli, Gregorios A. Paspatis); ${ }^{\circ}$ Hepatobiliary and Pancreatic Surgery Clinic, Metropolitan Hospital, Athens (Christos Dervenis); 'Department of Histopathology, Venizeleion General Hospital, Heraklion (George Kazamias, Elpida Giannikaki); ${ }^{\mathrm{d} D e p a r t m e n t ~ o f ~}$ Histopathology, Metropolitan Hospital, Athens (Athanasia Sepsa), Greece

Conflict of Interest: None

Correspondence to: Gregorios A. Paspatis, MD, PhD, Department of Gastroenterology, Venizeleion General Hospital, Knossou Avenue, 71409 Heraklion, Greece, e-mail: gpaspatis@gmail.com

Received 23 November 2020; accepted 11 December 2020; published online 26 February 2021

DOI: https://doi.org/10.20524/aog.2021.0610 over the previous 6 months. At admission, he was afebrile without any symptoms or signs of abdominal origin. Laboratory tests revealed normochromic, normocytic anemia (hemoglobin at $12.5 \mathrm{~g} / \mathrm{dL}$, normal range [NR] 13.4-17.4), and an elevation of $\gamma$-glutamyltransferase $(\gamma$-GT) $(202 \mathrm{U} / \mathrm{L}, \mathrm{NR} 0-50)$ and alkaline phosphatase (ALP) (184 U/L, NR 40-150). Magnetic resonance cholangiopancreatography (MRCP) demonstrated cholelithiasis, biliary sludge and a short stricture at the distal end of the CBD with upstream dilation $12 \mathrm{~mm}$ in diameter. The biliary tract was otherwise unremarkable. No pancreatic enlargement or narrowing of the main pancreatic duct was observed. An endoscopic retrograde cholangiopancreatography (ERCP) with sphincterotomy was performed. The presence of the above-mentioned CBD stricture was confirmed but no filling defects were observed. A straight plastic biliary stent (10 Fr, $7 \mathrm{~cm}$ ) was placed. The brush cytology obtained from the stricture was negative for malignancy.

The patient was admitted 6 months later, having undergone cholecystectomy, in order to undergo repeat ERCP for stent removal. In the meantime, he remained asymptomatic. Besides the known stricture, a hilar hepatic stricture was also demonstrated during the ERCP procedure. Cholangioscopy (SpyGlass $^{\mathrm{TM}}$, Boston Scientific, Marlborough, USA) revealed an irregular pattern, mucosal friability and anarchic vascularization (Fig. 1; Video 1) while targeted biopsies from the strictures showed non-specific signs of chronic inflammation. A new MRCP scan confirmed the ERCP findings (Fig.2). Quantification of serum immunoglobulins showed normal levels of IgG4 (76.9 mg/dL, NR 8-140). Viral serology, cancer antigen 19-9, $\alpha$-fetoprotein, carcinoembryonic antigen, antinuclear antibodies, antineutrophil cytoplasmic antibodies, anti-smooth muscle antibodies, and anti-mitochondrial antibodies were all negative.

In view of the persistent elevated levels of $\gamma$-GT and ALP, although the patient had experienced no symptoms, a new ERCP procedure was performed 2 months later. The cholangioscopic features of the strictures remained unchanged. 

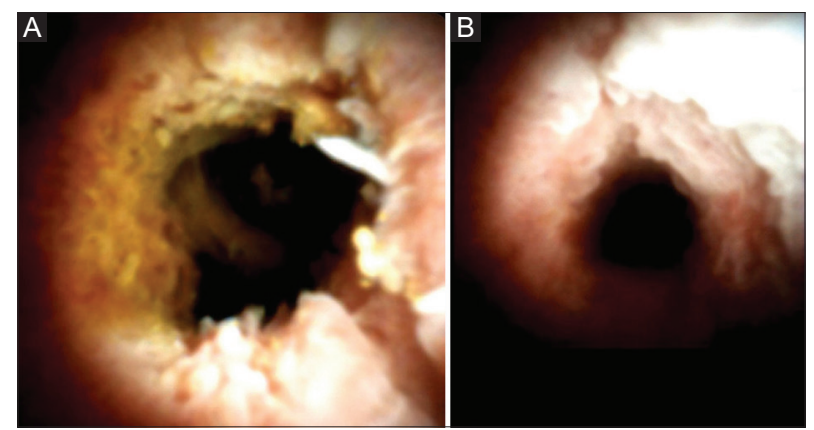

Figure 1 Cholangioscopic view of the (A) hilar hepatic stricture, and (B) common bile duct stricture, showing an irregular pattern, mucosal friability and anarchic vascularization (SpyGlass ${ }^{\mathrm{TM}}$, Boston Scientific)

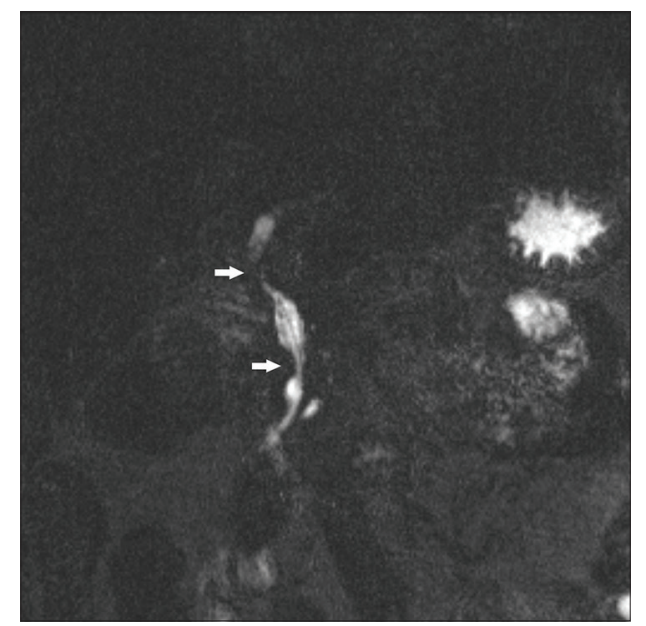

Figure 2 Magnetic resonance cholangiopancreatography demonstrated the presence of 2 segmental strictures at the hilar hepatic duct and at the distal end of the common bile duct (arrows). The straight plastic biliary stent, previously implanted during an endoscopic retrograde cholangiopancreatography procedure, can be seen in the common bile duct

Multiple targeted biopsies were again obtained and were negative for malignancy. A straight plastic biliary stent (11.5 Fr, $10 \mathrm{~cm}$ ) was positioned in the CBD. The patient's serum IgG4 levels were reexamined and remained within normal levels (81 mg/dL, NR 8-140). Based on the absence of elevated serum IgG4 concentrations, along with the non-involvement of other organs related with IgG4-RD, immunohistochemical stain for IgG4 on bile duct biopsies was not requested. In the following 3-month period, the patient was twice readmitted because of episodes of cholangitis and underwent 2 ERCP procedures. Both cholangiographic and cholangioscopic findings as well as histopathology of the lesions remained invariable (Fig.3; Video 2). A liver biopsy was performed with findings suggestive of non-specific cholestatic hepatitis.

We decided to suggest surgical treatment, given the high clinical suspicion of an underlying neoplasia (patient's weight loss, worrisome endoscopic appearance of the strictures, negative blood workup, recurrent episodes of cholangitis despite the presence of biliary stents). A hepaticojejunal anastomosis with lymphadenectomy was performed. Histopathology of
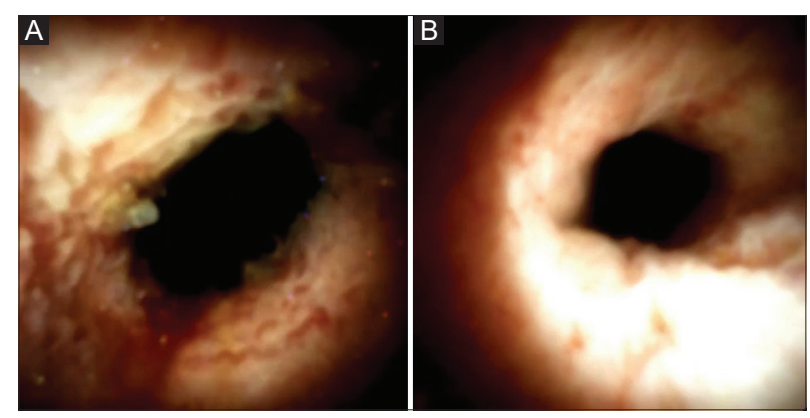

Figure 3 Cholangioscopic view of the (A) hilar hepatic stricture, and (B) common bile duct stricture 5 months after the initial cholangioscopy (SpyGlass $^{\mathrm{TM}}$, Boston Scientific)

the surgical specimen demonstrated lesions of IgG4-related SC with significant IgG4 infiltration of the CBD and regional lymph nodes. Cholestatic enzymes gradually normalized and the patient has been followed-up for 6 months without recurrence.

\section{Discussion}

IgG4-SC is frequently associated with AIP [3] and this association is useful for the diagnosis of IgG4-SC [4]. However, it is particularly difficult to diagnose IgG4-SC in the absence of AIP [5]. An elevation of serum IgG4 levels ( $\geq 140 \mathrm{mg} / \mathrm{dL})$ is seen in $90 \%$ of all IgG4-SC cases [6]. For type 3 IgG4-SC in particular, where cholangiographic and/or cholangioscopic findings tend to mimic cholangiocarcinoma, a cutoff of $\geq 206$ $\mathrm{mg} / \mathrm{dL}$ maybe useful for the diagnosis of IgG4-SC [6], since some cholangiocarcinomas exhibit high serum IgG4 levels. An even higher cutoff level of $560 \mathrm{mg} / \mathrm{dL}$ provides a more reliable marker for the differential diagnosis of IgG4-SC from malignancy [7]. In our case, serum IgG4 concentrations had been evaluated twice and histopathological examination of biopsy specimens did not reveal histologic findings indicative of IgG4-SC, namely, marked lymphoplasmacytic infiltration, storiform fibrosis and obliterative phlebitis. Immunohistochemical stain for IgG4 was not performed. However, in the absence of the aforementioned characteristic histologic findings, the diagnosis of IgG4-SC is not established based on IgG4 immunostaining alone [8]. A thorough diagnostic investigation of cholangiocarcinoma was conducted, including cholangioscopy-guided biopsies from the strictures. Taking into consideration that bile duct samples obtained with SpyGlass $^{\mathrm{Tu}}$ forceps (SpyBite ${ }^{\mathrm{TM}}$, Boston Scientific, Marlborough, USA) are often small, repeated biopsies were taken even though the reported sensitivity of cholangioscopyguided biopsies for malignant lesions is quite satisfactory (85\%) [9].

As an afterthought in this case, despite the fact that the diagnosis of IgG4-SC was not even possible according to the clinical diagnostic criteria for IgG4-SC [8], we could have evaluated the effectiveness of a steroid trial. Treatment with corticosteroids is effective in most IgG4-SC cases [8], although some malignant lesions may improve after steroid 
administration [10]. For the time being, our patient is being followed-up with laboratory evaluation every 6-8 weeks. In case of symptoms and/or signs of disease activity (abdominal pain, obstructive jaundice, elevated serum hepatobiliary enzyme levels, new biliary strictures), steroid therapy will be initiated.

In conclusion, this case shows that IgG4-SC should always be considered as part of the differential diagnosis in patients presenting with unclassified intrahepatic and/or extrahepatic biliary strictures, even in the absence of elevated serum IgG4 levels when a thorough work up for malignancy is negative. Our case report highlights the fact that distinguishing IgG4SC from cholangiocarcinoma may occasionally be remarkably difficult on a clinical basis.

Video 1 Cholangioscopy demonstrated the presence of 2 segmental strictures at the hilar hepatic and common bile duct with irregular pattern, friability and anarchic vasculatization

You can see https://youtu.be/X3c_KO8_xx8

Video 2 Cholangioscopy performed 5 months after the initial one indicated that the features of the strictures remained unchanged You can see https://youtu.be/BTCHiF2C5Vw

\section{References}

1. Kamisawa T, Zen Y, Pillai S, Stone JH. IgG4-related disease. Lancet 2015;385:1460-1471.
2. Deshpande V, Zen Y, Chan JK, et al. Consensus statement on the pathology of IgG4-related disease. Mod Pathol 2012;25:1181-1192.

3. Ghazale A, Chari ST, Zhang L, et al. Immunoglobulin G4associated cholangitis: clinical profile and response to therapy. Gastroenterology 2008;134:706-715.

4. Nakazawa T, Naitoh I, Hayashi K, et al. Diagnostic criteria for IgG4-related sclerosing cholangitis based on cholangiographic classification. J Gastroenterol 2012;47:79-87.

5. Graham RP, Smyrk TC, Chari ST, Takahashi N, Zhang L. Isolated IgG4-related sclerosing cholangitis: a report of 9 cases. Hum Pathol 2014;45:1722-1729.

6. Ohara H, Nakazawa T, Kawa S, et al. Establishment of a serum IgG4 cut-off value for the differential diagnosis of IgG4-related sclerosing cholangitis: a Japanese cohort. J Gastroenterol Hepatol 2013;28:1247-1251.

7. Oseini AM, Chaiteerakij R, Shire AM, et al. Utility of serum immunoglobulin G4 in distinguishing immunoglobulin G4associated cholangitis from cholangiocarcinoma. Hepatology 2011;54:940-948.

8. Ohara H, Okazaki K, Tsubouchi H, et al; Japan Biliary Association. Clinical diagnostic criteria of IgG4-related sclerosing cholangitis 2012. J Hepatobiliary Pancreat Sci 2012;19:536-542.

9. Navaneethan U, Hasan MK, Kommaraju K, et al. Digital, single-operator cholangiopancreatoscopy in the diagnosis and management of pancreatobiliary disorders: a multicenter clinical experience (with video). Gastrointest Endosc 2016;84:649-655.

10. Tomiyama T, Uchida K, Matsushita M, et al. Comparison of steroid pulse therapy and conventional oral steroid therapy as initial treatment for autoimmune pancreatitis. J Gastroenterol 2011;46:696-704. 